\title{
Qualidade de água dos rios Vacacaí e Vacacaí-Mirim no Estado do Rio Grande do Sul, Brasil
}

\author{
Water quality of the Vacacaí and Vacacaí-Mirim rivers in the Rio Grande do Sul State, Brazil
}

\author{
Enio Marchesan ${ }^{I *}$ Gerson Meneghetti Sarzi Sartori ${ }^{\mathrm{I}}$ Geovane Boschmann Reimche ${ }^{\mathrm{IV}}$ \\ Luis Antonio de AvilaI Renato ZanellaI ${ }^{\text {II }}$ Sérgio Luiz de Oliveira Machado ${ }^{\text {IV }}$ \\ Vera Regina Mussoi Macedo ${ }^{\mathrm{III}}$ Juliana Pivetta Cogo ${ }^{\mathrm{II}}$
}

\section{RESUMO}

A água é um dos recursos naturais fundamentais para a vida. Neste trabalho, foi avaliada a qualidade da água dos rios Vacacai e Vacacai-Mirim no Rio Grande do Sul (RS), Brasil, com base nos parâmetros $\mathrm{N}$-nitrato $\left(\mathrm{N}-\mathrm{NO} \mathrm{O}_{3}\right), \mathrm{N}$-amônia $\left(\mathrm{N}-\mathrm{NH}_{3}\right)$, fósforo $(\mathrm{P})$, potássio $(\mathrm{K})$ e sódio $(\mathrm{Na})$ e também na variação do $\mathrm{pH}$ e da condutividade elétrica (CE) da água. As coletas de água foram realizadas a cada 15 dias, de outubro a março de 2002/03 a 2006/07. Os resultados foram comparados com as legislações do RS (Secretaria da Saúde e Meio Ambiente), do Brasil (CONAMA e Ministério da Saúde) e dos Estados Unidos (EPA). O pH variou de 5,71 a 7,50, e a CE, de 33,33 a 118,88 $\mu \mathrm{S} \mathrm{cm}^{-1}$. Para $N$-nitrato, as amostras de água estiveram dentro de limites aceitáveis estabelecidos pelas legislações. A concentração de $P$ oscilou de 0,00 a $0,18 m g L^{-1}$. Os níveis de $N$-amônia variaram de 0,02 a $0,39 m g L^{-1}$, o $K$, de 2,05 a 3,66mg $L^{-1}$ e o $\mathrm{Na}$, de 1,84 a 16,8mg $\mathrm{L}^{-1}$.

Palavras-chave: bacia hidrográfica, nutrientes, contaminação ambiental, sustentabilidade.

\section{ABSTRACT}

Water is an important natural resource for preserving the life in the planet. In this survey, it was evaluated water quality in the Vacacai and Vacacai-Mirim basins in Rio Grande do Sul State, Brazil. The water samples were evaluated for $\mathrm{N}$-nitrate $\left(\mathrm{N}-\mathrm{NO}_{3}\right)$, phosphorus $(\mathrm{P})$, potassium $(\mathrm{K})$ and sodium (Na), $\mathrm{pH}$ and electric conductivity (EC). The results were compared to the values suggested by the resolutions of the State Environmental Agency (Secretaria da Saude e Meio Ambiente), the Brazilian Agencies (CONAMA e Ministério da
Saúde) and the United States Environmental Protection Agency (EPA). The water pH varied between 5.71 and 7.5 and de EC between 33.33 and $118.88 \mu \mathrm{S} \mathrm{cm}^{-1}$. For $N$-nitrate 100\% samples were in the settled limits by environmental legislation. The $P$ levels were between 0.00 to $0.18 \mathrm{mg} L^{-1}$. The $N$-ammonia levels varied between 0.02 and $0.39 \mathrm{mg} \mathrm{L}^{-1}$, the $K$ levels remained between 2.05 and $3.66 \mathrm{mg} \mathrm{L}^{-1}$ and the Na levels between 1.84 and $16.8 \mathrm{mg} \mathrm{L}^{-1}$.

Key words: hydrographic basin, nutrients, environmental contamination, sustainability.

\section{INTRODUÇÃo}

A degradação dos recursos naturais e a contaminação da água por fertilizantes e outros químicos vem crescendo e trazendo graves conseqüências para o ambiente e para a saúde humana. O crescimento da atividade agropecuária e a perda de sedimentos por meio do escoamento superficial afetam a qualidade das águas superficiais não apenas no local de origem da contaminação, mas também em outros pontos de interferência dos recursos hídricos.

O risco de contaminação é particularmente importante em explorações agropecuárias a jusante das bacias hidrográficas, pois as concentrações de $\mathrm{P}$ e $\mathrm{N}$ geralmente excedem níveis detectados em áreas não agrícolas (HAMER et al., 2004; CHAU, 2007), sendo a

'Departamento de Fitotecnia, Centro de Ciências Rurais (CCR), Universidade Federal de Santa Maria (UFSM), 97105-900, Santa Maria, RS, Brasil. E-mail: emarchezan@terra.com.br. *Autor para correspondência.

"Laboratório de Análises de Resíduos de Pesticidas (LARP), Centro de Ciências Naturais e Exatas (CCNE), UFSM, Santa Maria, RS, Brasil.

IIIInstituto Rio Grandense do Arroz (IRGA), EEA, Cachoeirinha, RS, Brasil.

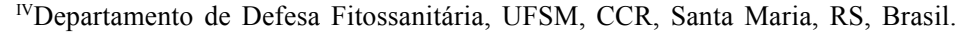


eutrofização um dos maiores problemas na qualidade da água. De acordo com PIONKE et al. (2000), cerca de $33 \%$ das áreas agrícolas são responsáveis por $58 \%$ do $\mathrm{P}$ que chega aos mananciais hídricos. Em água doce, reações do $\mathrm{N}$-nitrato podem causar depleção de oxigênio, afetando os organismos aquáticos aeróbicos. A N-amônia é altamente tóxica e, mesmo em concentrações baixas, pode afetar a sobrevivência de peixes (SUNDARAY et al., 2005).

Analisando os teores de nutrientes e outros parâmetros químicos de águas de açudes, lagoas e rios da região Sul do Brasil, DIEL et al. (2007) encontraram valores médios de $99,41,963$ e $5760 \mathrm{mg} \mathrm{L}^{-1}$, respectivamente, para $\mathrm{N}$-nitrato, fósforo $(\mathrm{P})$, potássio $(\mathrm{K})$ e sódio $(\mathrm{Na}) ; 116 \mu \mathrm{S} \mathrm{cm}^{-1}$ para condutividade elétrica (CE) e 6,8 para pH. Em Santa Catarina, em estudo na microbacia do Ribeirão Fortuna, PINHEIRO \& DESCHAMPS (2008) concluíram que as cargas anuais transportadas de ortofosfato e nitrato variam de 6,51 a 62,79 e de 1,13 a $4,97 \mathrm{~kg} \mathrm{ha}^{-1}$ ano $^{-1}$, respectivamente. Nos Estados Unidos, CARPENTER et al. (1998) concluíram que 82 e $84 \%$ dos descartes de N-total e Ptotal, respectivamente, nas águas superficiais, são provenientes de fontes difusas e que, acima de $90 \%$ destes, são de áreas rurais. $\mathrm{O}$ pH exerce efeito direto sobre os ecossistemas aquáticos naturais pela alteração da fisiologia das espécies (MARQUES et al., 2007), enquanto o efeito indireto influencia a precipitação de elementos químicos pesados e a solubilidade de nutrientes (PIVELI \& KATO, 2006). ACE é um indicativo da quantidade de sólidos em suspensão na água, pois, segundo CARVALHO et al. (2000), o aumento de sólidos suspensos na água favorece a condutividade elétrica no ambiente.

Diante do papel da água para a vida e da magnitude dos efeitos da sua deteriorização sobre as atividades humanas, torna-se importante o monitoramento da composição química e da qualidade de água de rios próximos a sistemas de produção agrícola. Nesse sentido, o presente estudo teve por objetivo avaliar a qualidade de água com base nos parâmetros de N-nitrato, N-amônia, $\mathrm{P}, \mathrm{K}, \mathrm{Na}$ e também na variação do $\mathrm{pH}$ e da $\mathrm{CE}$, nas águas dos rios Vacacaí e Vacacaí-Mirim, em cinco safras agrícolas, durante o período estival de desenvolvimento das culturas.

\section{MATERIAL E MÉTODOS}

As bacias hidrográficas dos rios Vacacaí e Vacacaí-Mirim estão localizadas na Depressão Central do Rio Grande do Sul (RS), situadas entre as coordenadas geográficas de $29^{\circ} 35^{\prime}$ a $30^{\circ} 45^{\prime}$ de latitude sul; e $53^{\circ} 04^{\prime}$ a $54^{\circ} 34^{\prime}$ de longitude oeste, possuindo uma área de total de $11.085,77 \mathrm{~km}^{2}$ e abrangendo uma população total de 407.533 habitantes (RS, 2007).
As bacias hidrográficas foram monitoradas durante os meses de outubro a março, período de cultivos mais importantes, nas safras agrícolas de 2002/ 03 a 2006/07, na Depressão Central do RS, Brasil, totalizando 120 amostras de água por ano, sendo 60 amostras em cada um dos rios. As coletas foram realizadas quinzenalmente, em 10 pontos de cada rio, e estes constituíram as repetições e foram escolhidos pela representatividade quanto à descarga de água das lavouras para os rios. Os tratamentos fatoriais avaliados foram as safras (2002/03 a 2006/07) e os meses de amostragem (outubro a março). A maioria das áreas das bacias hidrográficas desses rios é ocupada com explorações agrícolas e, no caso do arroz, conduzidas com lâmina de água contínua após a irrigação.

Para a coleta das amostras, utilizou-se uma garrafa de politereftalato de etila com capacidade de dois litros, perfurada da metade até a extremidade superior e acoplada a um suporte com peso para que afundasse na vertical, em diferentes profundidades, à qual foi amarrada uma corda para facilitar o seu lançamento e a retirada da água.

A água amostrada foi transferida para dois frascos de vidro (cor âmbar) de $500 \mathrm{~mL}$, um deles já contendo $2 \mathrm{~mL}$ de $\mathrm{H}_{2} \mathrm{SO}_{4}$ a $2,5 \mathrm{~mol} \mathrm{~L}^{-1}$, para acidificação da amostra $(\mathrm{pH}<2, \mathrm{O})$, evitando com isso perdas de nitrogênio. Esses frascos foram conservados em caixa de isopor, com gelo, durante o seu transporte até o Laboratório de Análise de Resíduos de Pesticidas (LARP) do Departamento de Química da Universidade Federal de Santa Maria (UFSM). Nesse laboratório, as amostras acidificadas foram armazenadas em geladeira e, um dia após cada coleta, analisadas pelo método espectrofotométrico $\left(4500 \mathrm{NO}_{3} \mathrm{E}\right)$, para nitrato, e pelo método espectrofotométrico (4500- $\left.\mathrm{NH}_{3} \mathrm{C}\right)$, para amônia, de acordo com (APHA, 1995).

$\mathrm{O} \mathrm{pH}$ e a CE da água foram determinados em alíquotas das amostras não acidificadas, sem nenhum tratamento prévio (TEDESCO et al., 1995). O restante destas amostras foi filtrado em papel JP n.42 e armazenado à temperatura de $3^{\circ} \mathrm{C}$. Para as determinações de $\mathrm{P}, \mathrm{K}$ e Na, uma alíquota de $100 \mathrm{~mL}$ das amostras filtradas foi clarificada com $2 \mathrm{~mL}$ de $\mathrm{HCl}(50 \%)$. O K e o $\mathrm{Na}$ foram determinados por espectrometria de absorção atômica (3500 B). A determinação do P foi realizada por espectrofotometria do molibdovanadofosfato (4500 P) para $\mathrm{P}\left(\mathrm{PO}_{4}^{-3}\right)$ de acordo com APHA (1995).

Os resultados obtidos foram submetidos às análises das pressuposições do modelo matemático e, em seguida, foi realizada a análise de variância com teste F. Cumprindo os pressupostos, as médias foram com comparadas pelo teste de Tukey, a 5\% de probabilidade de erro. 


\section{RESULTADOS E DISCUSSÃO}

Os resultados da composição química das águas dos rios Vacacaí e Vacacaí-Mirim são apresentados nas tabelas $1 \mathrm{e} 2$. Para definir a qualidade da água de mananciais hídricos, eles são enquadrados em classes de acordo com a Resolução CONAMA n.357 (BRASIL, 2005). A concentração dos nutrientes analisados variou entre os rios, fato também observado por DIEL et al. (2007), para outros rios da região Sul do RS. As concentrações de $\mathrm{N}^{-\mathrm{NO}_{3}}$ variaram de 0,02 a $4,96 \mathrm{mg} \mathrm{L}^{-1}$ (rio Vacacaí) e de 0,03 a $5,71 \mathrm{mg} \mathrm{L}^{-1}$ (rio Vacacaí-Mirim), encontrando-se abaixo da concentração máxima permitida para água de rios, que é de $10 \mathrm{mg} \mathrm{L}^{-1}$ (RS, 1989; BRASIL, 2005; EPA, 2008). Para N-NH concentrações variaram de $0,02 \mathrm{a} 0,39 \mathrm{mg} \mathrm{L}^{-1}$ (rio Vacacaí) e de 0,02 a $0,18 \mathrm{mg} \mathrm{L}^{-1}$ (rio Vacacaí-Mirim), portanto inferiores ao limite permitido para águas de classe I, que é de $3,7 \mathrm{mg} \mathrm{L}^{-1}$, para $\mathrm{pH}=7,5$, estabelecido pela Resolução CONAMA n.357 (BRASIL, 2005) e de 1,5mg $\mathrm{L}^{-1}$ estabelecido pela Portaria 518 do Ministério da Saúde (BRASIL, 2004). Em ambos os rios avaliados, foi possível verificar que a safra 2002/03 (Tabela 1 e 2) apresentou o período de maior concentração de $\mathrm{N}_{-} \mathrm{NO}_{3}$ e $\mathrm{N}-\mathrm{NH}_{3}$, podendo ser decorrente da elevada precipitação pluvial ocorrida, o que contribuiu para o maior carreamento de nutrientes nos rios. $\mathrm{O}$ aporte médio de $\mathrm{N}_{-} \mathrm{NO}_{3}$ encontrado neste trabalho foi inferior ao obtido por DIEL et al. (2007) para outros rios da região Sul do RS, que variou de 25 a $421 \mathrm{mg} \mathrm{L}^{-1}$. As concentrações mais altas de $\mathrm{N}$ podem estar relacionadas à descarga de dejetos de efluentes urbanos e industriais e ao aporte de $\mathrm{N}$ transferido pela água da chuva de lavouras agrícolas existentes na bacia hidrográfica desses rios ou pela drenagem da água de lavouras de arroz irrigado lançada em seus cursos.

Do total de amostras de água analisadas, 90\% do Rio Vacacaí e $93 \%$ do rio Vacacaí-Mirim apresentaram concentração de $\mathrm{P}$ abaixo do máximo estabelecido por RS (1989), que é de $0,1 \mathrm{mg} \mathrm{L}^{-1}$, permitindo enquadrá-las nas classes I e II. No entanto, de acordo com os limites estabelecidos pela Resolução CONAMA n.357 (BRASIL, 2005), 3,3\% das amostras apresentaram valores de $\mathrm{P}$ abaixo de $0,15 \mathrm{mg} \mathrm{L}^{-1}$, enquadrando-se na classe III. Por essa mesma resolução, apenas $6,6 \%$ das amostras de água do rio Vacacaí e 3,3\% do rio Vacacaí-Mirim ultrapassam o limite para classe III. As concentrações mais altas de P na água foram de $0,18 \mathrm{mg} \mathrm{L}^{-1}$ (rio Vacacaí) e $0,16 \mathrm{mg} \mathrm{L}^{-1}$ (rio Vacacaí-Mirim) em 2002/03. Essas concentrações podem estar associadas às mesmas hipóteses levantadas para o N. Além disso, o P geralmente é transportado através do fluxo superficial por ser fortemente adsorvido pelos colóides do solo, o que o torna pouco móvel. Assim, esse elemento tende a se acumular na posição em que é aplicado nos cultivos agrícolas, sendo transferido de local quando a porção do solo em que se encontra sofre movimentação (CORREL, 1988; GINTING et al., 1998).

A concentração média de $\mathrm{K}$, nas amostras de água do rio Vacacaí, variou de 2,05 a 2,96 $\mathrm{mg} \mathrm{L}^{-1} \mathrm{e}$, no rio Vacacaí-Mirim, de 3,01 a 3,66 $\mathrm{mg} \mathrm{L}^{-1}$ ( Tabelas 1 e 2). Em 2005/06, as concentrações de K, nas amostras de água, foram maiores que as demais safras, notadamente a partir de novembro; e variaram de 3,35 a $7,35 \mathrm{mg} \mathrm{L}^{-1}$ (rio Vacacaí) e de 5,89 a 8,40 $\mathrm{mg} \mathrm{L}^{-1}$ (rio Vacacaí-Mirim). A maior concentração de $\mathrm{K}$, nas amostras de água, pode ser explicada, em parte, pela dinâmica desse nutriente, que é mais solúvel e móvel no solo, favorecendo o transporte e a lixiviação (BERTOL et al., 2004; DIEL et al., 2007).

Para $\mathrm{Na}, 100 \%$ das concentrações, nas amostras de água, estiveram abaixo do limite para água destinada ao consumo humano, que é de $200 \mathrm{mg} \mathrm{L}^{-1}$ (BRASIL, 2004). No rio Vacacaí, as concentrações variaram de 1,84 a $16,81 \mathrm{mg} \mathrm{L}^{-1}$, concentrações próximas ao rio Vacacaí-Mirim, que variou de 2,41 a 14,81 $\mathrm{mg} \mathrm{L}^{-1}$. De forma semelhante ao que ocorreu com o K, o Na apresentou elevadas concentrações na safra 2005/06. Segundo ODEMIS \& EVRENDILEK (2007), o aumento da concentração de íons pode ser atribuído à lixiviação de fertilizantes químicos pela precipitação, que podem ser oriundos de diferentes fontes, como fertilizantes agrícolas, industriais e resíduos domésticos.

Essa variabilidade de concentrações entre os nutrientes analisados pode ser decorrente de inúmeros fatores, dentre eles, a precipitação pluvial (Figura 1) e a mobilidade do elemento mineral no solo, que é baixa para o $\mathrm{P}$ e alta para $\mathrm{N}$ e $\mathrm{K}$. Com isso, as perdas podem ocorrer em pontos localizados (P) ou na maior parte da bacia hidrográfica $(\mathrm{N} \mathrm{e} \mathrm{K})$.

Entre os rios, houve pequena diferença no pH da água; 73\% (Rio Vacacaí) e 76\% (Rio VacacaíMirim) das amostras de água se enquadraram na faixa estabelecida pela Resolução CONAMA n ${ }^{\circ} 357$ (BRASIL, 2005), para as águas de superfície classe 1 (níveis de 6 a 9). Pela Portaria $05 / 89$ da Secretaria da Saúde e Meio Ambiente do RS (1989), 73\% (rio Vacacaí) e 77\% (rio Vacacaí-Mirim) das amostras de água mantiveram níveis de $\mathrm{pH}$ dentro da faixa estabelecida $(6$ a 8,5). Pela EPA (2008), apenas 40\% (Rio Vacacaí) e 37\% (Rio VacacaíMirim) das amostras de água mantiveram os níveis de $\mathrm{pH}$ dentro da faixa permitida (6,5 a 8,5). Em geral, os valores médios de $\mathrm{pH}$ estão dentro da faixa encontrada por DIEL et al. (2007) em águas de diferentes rios da região Sul do RS. O pH não apresentou variações tão 
Tabela 1 - Concentrações médias de nutrientes, pH e CE na água do rio Vacacaí. Santa Maria, RS. 2009.

\begin{tabular}{|c|c|c|c|c|c|c|}
\hline Safras & outubro & novembro & $\begin{array}{l}\text { dezembro } \\
\text { de--Amo }\end{array}$ & $\begin{array}{l}\text { ns------------- } \\
\text { janeiro }\end{array}$ & fevereiro & março \\
\hline & \multicolumn{6}{|c|}{$\mathrm{N}-\mathrm{NO}_{3}\left(\mathrm{mg} \mathrm{L}^{-1}\right)$} \\
\hline $2002 / 03$ & $\mathrm{CD} 0,54 \mathrm{ab}$ & $\mathrm{BC} 1,63 \mathrm{a}$ & A 3,42 a & D $0,29 \mathrm{~b}$ & $\mathrm{AB} 3,03 \mathrm{a}$ & D $0,49 \mathrm{bc}$ \\
\hline $2003 / 04$ & $\mathrm{BC} 0,52 \mathrm{ab}$ & $\mathrm{AB} 0,75 \mathrm{ab}$ & $\mathrm{BC} 0,46 \mathrm{~b}$ & $\mathrm{BC} 0,50 \mathrm{~b}$ & A $2,53 \mathrm{ab}$ & $\mathrm{C} 0,02 \mathrm{c}$ \\
\hline $2004 / 05$ & NS 0,84 a & $0,52 \mathrm{~b}$ & $0,61 \mathrm{~b}$ & $0,66 \mathrm{~b}$ & $1,10 \mathrm{bc}$ & $0,84 \mathrm{~b}$ \\
\hline $2005 / 06$ & D $0,16 \mathrm{~b}$ & D $0,19 b$ & $\mathrm{CD} 0,72 \mathrm{~b}$ & B 2,14 a & $\mathrm{BC} 1,66 \mathrm{ab}$ & A 4,96 a \\
\hline $2006 / 07$ & NS $0,62 \mathrm{ab}$ & $0,46 \mathrm{~b}$ & $0,59 \mathrm{~b}$ & $0,53 \mathrm{~b}$ & $0,43 \mathrm{c}$ & $0,45 \mathrm{bc}$ \\
\hline Média & 0,53 & 0,71 & 1,16 & 0,82 & 1,75 & 1,35 \\
\hline CV $(\%)$ & \multicolumn{6}{|c|}{30,39} \\
\hline $2002 / 03$ & B $0,07 \mathrm{~ns}$ & B $0,07 \mathrm{~ns}$ & $\mathrm{AB} 0,14 \mathrm{a}$ & B $0,08 \mathrm{~ns}$ & A $0,21 \mathrm{a}$ & B $0,06 \mathrm{~b}$ \\
\hline $2003 / 04$ & B 0,07 & B 0,07 & B $0,06 \mathrm{ab}$ & B 0,04 & B $0,04 \mathrm{~b}$ & A 0,39 a \\
\hline $2004 / 05$ & B 0,09 & B 0,08 & B $0,11 \mathrm{a}$ & B 0,07 & B $0,06 \mathrm{~b}$ & A 0,33 a \\
\hline $2005 / 06$ & NS 0,04 & 0,02 & $0,02 \mathrm{~b}$ & 0,03 & $0,02 \mathrm{~b}$ & $0,02 \mathrm{~b}$ \\
\hline $2006 / 07$ & NS 0,08 & 0,05 & $0,07 \mathrm{ab}$ & 0,05 & $0,06 \mathrm{~b}$ & $0,05 \mathrm{~b}$ \\
\hline Média & 0,07 & 0,06 & 0,08 & 0,05 & 0,08 & 0,17 \\
\hline CV (\%) & \multicolumn{6}{|c|}{22,42} \\
\hline $2002 / 03$ & 0,04 & 0,16 & 0,14 & 0,05 & 0,18 & $0,00^{1}$ \\
\hline $2003 / 04$ & 0,06 & 0,01 & 0,01 & 0,01 & 0,08 & $0,00^{1}$ \\
\hline $2004 / 05$ & 0,01 & 0,03 & $0,00^{1}$ & 0,01 & 0,01 & $0,00^{1}$ \\
\hline $2005 / 06$ & 0,02 & 0,01 & 0,03 & 0,03 & 0,03 & 0,01 \\
\hline $2006 / 07$ & 0,07 & 0,08 & 0,07 & 0,07 & 0,08 & 0,09 \\
\hline Média & 0,04 & 0,06 & 0,05 & 0,03 & 0,08 & 0,02 \\
\hline CV (\%) & \multicolumn{6}{|c|}{22,42} \\
\hline $2002 / 03$ & A $2,33 \mathrm{ab}$ & $\mathrm{ABC} 1,43 \mathrm{~b}$ & $\mathrm{C} 0,95 \mathrm{~b}$ & $\mathrm{BC} 1,17 \mathrm{~b}$ & $\mathrm{ABC} 1,69 \mathrm{bc}$ & $\mathrm{AB} 2,20 \mathrm{~b}$ \\
\hline $2003 / 04$ & NS $1,70 \mathrm{~b}$ & $2,06 \mathrm{~b}$ & $1,20 \mathrm{~b}$ & $1,28 \mathrm{~b}$ & $1,20 \mathrm{c}$ & $1,04 \mathrm{c}$ \\
\hline $2004 / 05$ & NS $2,37 \mathrm{ab}$ & $1,70 \mathrm{~b}$ & $1,69 \mathrm{~b}$ & $1,75 \mathrm{~b}$ & $2,64 \mathrm{~b}$ & $2,68 \mathrm{~b}$ \\
\hline $2005 / 06$ & C $3,35 \mathrm{a}$ & C $3,47 \mathrm{a}$ & B $4,70 \mathrm{a}$ & B $4,85 \mathrm{a}$ & A 7,37 a & A 6,69 a \\
\hline $2006 / 07$ & NS $1,92 \mathrm{~b}$ & $1,60 \mathrm{~b}$ & $1,83 \mathrm{~b}$ & $1,71 \mathrm{~b}$ & $1,28 \mathrm{c}$ & $2,20 \mathrm{~b}$ \\
\hline Média & 2,33 & 2,05 & 2,07 & 2,40 & 2,84 & 2,96 \\
\hline CV $(\%)$ & \multicolumn{6}{|c|}{24,46} \\
\hline $2002 / 03$ & A 6,56 b & B 3,36 bc & B $3,70 \mathrm{~b}$ & $\mathrm{AB} 4,00 \mathrm{~b}$ & A $6,48 \mathrm{~b}$ & B $2,75 \mathrm{~b}$ \\
\hline $2003 / 04$ & A $4,83 \mathrm{~b}$ & A $5,88 \mathrm{~b}$ & B $1,84 \mathrm{~b}$ & $\mathrm{AB} 4,21 \mathrm{~b}$ & $\mathrm{AB} 4,63 \mathrm{~b}$ & A $5,26 \mathrm{~b}$ \\
\hline $2004 / 05$ & NS 5,14 b & $4,25 \mathrm{ab}$ & $3,06 \mathrm{~b}$ & $3,75 \mathrm{~b}$ & $4,66 \mathrm{~b}$ & $4,76 \mathrm{~b}$ \\
\hline $2005 / 06$ & B $11,52 \mathrm{a}$ & B $12,71 \mathrm{a}$ & A $16,81 \mathrm{a}$ & B 12,36 a & B $11,95 \mathrm{a}$ & B $12,52 \mathrm{a}$ \\
\hline $2006 / 07$ & NS $4,76 \mathrm{~b}$ & $3,10 \mathrm{c}$ & $3,31 \mathrm{~b}$ & $4,19 \mathrm{~b}$ & $4,10 \mathrm{~b}$ & $4,72 \mathrm{~b}$ \\
\hline Média & 6,56 & 5,86 & 5,74 & 6,07 & 6,90 & 6,00 \\
\hline $\mathrm{CV}(\%)$ & \multicolumn{6}{|c|}{25,16} \\
\hline $2002 / 03$ & $\mathrm{BC} 6,21 \mathrm{bc}$ & D $5,75 \mathrm{c}$ & B $6,25 \mathrm{~b}$ & B $6,44 \mathrm{~b}$ & A $6,77 \mathrm{c}$ & CD 5,94 d \\
\hline $2003 / 04$ & $\mathrm{ABC} 5,96 \mathrm{c}$ & C $5,71 \mathrm{c}$ & BC $5,80 \mathrm{c}$ & A 6,21 bc & $\mathrm{AB} 6,06 \mathrm{~d}$ & A $6,23 \mathrm{c}$ \\
\hline $2004 / 05$ & B 5,65 d & $\mathrm{AB} 5,93 \mathrm{c}$ & B $5,69 \mathrm{c}$ & A $6,06 \mathrm{c}$ & A $6,09 \mathrm{~d}$ & A $6,06 \mathrm{~cd}$ \\
\hline $2005 / 06$ & C $6,44 \mathrm{~b}$ & B $6,84 \mathrm{a}$ & B $6,79 \mathrm{a}$ & B 7,01 a & A 7,50 a & $\mathrm{BC} 6,73 \mathrm{~b}$ \\
\hline $2006 / 07$ & $\mathrm{BC} 6,78 \mathrm{a}$ & $\mathrm{C} 6,52 \mathrm{~b}$ & $\mathrm{BC} 6,79 \mathrm{a}$ & $\mathrm{AB} 6,89 \mathrm{a}$ & A 7,12 b & $\mathrm{AB} 7,04 \mathrm{a}$ \\
\hline Média & 6,21 & 6,15 & 6,26 & 6,52 & 6,71 & 6,40 \\
\hline CV $(\%)$ & \multicolumn{6}{|c|}{2,56} \\
\hline $2002 / 03$ & $\mathrm{AB} 75,25 \mathrm{ab}$ & $\mathrm{C} 41,60 \mathrm{c}$ & $\mathrm{ABC} 58,44 \mathrm{~b}$ & $\mathrm{ABC} 59,54 \mathrm{c}$ & A $80,46 \mathrm{~ns}$ & BC $50,78 \mathrm{~b}$ \\
\hline $2003 / 04$ & A $61,04 \mathrm{~b}$ & A $80,30 \mathrm{ab}$ & B $33,33 \mathrm{c}$ & A 68,66 bc & A 77,62 & A 79,76 a \\
\hline $2004 / 05$ & A 88,98 a & B 59,26 bc & B $57,30 \mathrm{bc}$ & $\mathrm{AB} 68,85 \mathrm{bc}$ & $\mathrm{AB} 75,74$ & $\mathrm{AB} 72,98 \mathrm{ab}$ \\
\hline $2005 / 06$ & $\mathrm{BC} 83,32 \mathrm{ab}$ & $\mathrm{ABC} 93,22 \mathrm{a}$ & A $115,51 \mathrm{a}$ & BC $85,80 \mathrm{ab}$ & C 68,67 & $\mathrm{AB} 95,36 \mathrm{a}$ \\
\hline $2006 / 07$ & B $67,68 \mathrm{ab}$ & A 102,38 a & A $104,07 \mathrm{a}$ & A $95,11 \mathrm{a}$ & AB 91,92 & A $94,78 \mathrm{a}$ \\
\hline Média & 5,25 & 75,35 & 73,73 & 75,60 & 8,88 & 78,73 \\
\hline CV (\%) & \multicolumn{6}{|c|}{18,42} \\
\hline
\end{tabular}

*Nas linhas, médias não antecedidas da mesma letra maiúscula diferem entre meses; e, nas colunas, médias não seguidas da mesma letra minúscula diferem dos anos pelo teste de Tukey $(\mathrm{P}=0,05)$.

$\left(^{1}\right)$ Não detectado: $<0,006 \mathrm{mg} \mathrm{L}^{-1}$ de $\mathrm{P}$.

${ }_{\text {ns }}$ Teste $\mathrm{F}$ não significativo $(\mathrm{P}=0,05)$. 
Tabela 2 - Concentrações médias de nutrientes, pH e CE na água do rio Vacacaí-Mirim. Santa Maria, RS. 2009.

\begin{tabular}{|c|c|c|c|c|c|c|}
\hline Safras & outubro & novembro & dezembro & $\begin{array}{l}\text { ns-------------- } \\
\text { janeiro }\end{array}$ & fevereiro & março \\
\hline & \multicolumn{6}{|c|}{ 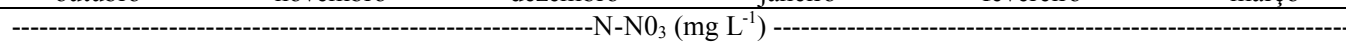 } \\
\hline $2002 / 03$ & $\mathrm{AB} 3,15 \mathrm{a}^{*}$ & BC $2,83 \mathrm{~ns}$ & BC 2,44 ns & A 4,17 a & $\mathrm{AB} 2,79 \mathrm{a}$ & $\mathrm{C} 1,17 \mathrm{~b}$ \\
\hline $2003 / 04$ & A $1,14 \mathrm{~b}$ & A 1,31 & A 1,28 & A $0,86 \mathrm{c}$ & A 0,95 b & B $0,03 \mathrm{c}$ \\
\hline $2004 / 05$ & NS $1,53 \mathrm{~b}$ & 1,53 & 1,72 & $1,56 \mathrm{bc}$ & $1,33 \mathrm{~b}$ & $1,19 \mathrm{~b}$ \\
\hline $2005 / 06$ & $\mathrm{BC} 2,00 \mathrm{ab}$ & BC 1,93 & C 1,55 & BC 2,03 b & B 3,12 a & A 5,71 a \\
\hline $2006 / 07$ & NS $1,20 \mathrm{~b}$ & 1,22 & 1,32 & $1,24 b c$ & $1,03 \mathrm{~b}$ & $1,16 \mathrm{~b}$ \\
\hline Média & 1,80 & 1,76 & 1,66 & 1,97 & 1,84 & 1,85 \\
\hline CV $(\%)$ & \multicolumn{6}{|c|}{20,75} \\
\hline $2002 / 03$ & $\mathrm{CD} 0,07 \mathrm{ab}$ & $\mathrm{AB} 0,14 \mathrm{a}$ & $\mathrm{BC} 0,09 \mathrm{a}$ & A 0,18 a & $\mathrm{D} 0,03 \mathrm{~b}$ & CD 0,06 ab \\
\hline $2003 / 04$ & B 0,06 bc & $\mathrm{AB} 0,08 \mathrm{~b}$ & B $0,04 \mathrm{ab}$ & B $0,07 \mathrm{bc}$ & A 0,13 a & $\mathrm{AB} 0,08 \mathrm{a}$ \\
\hline $2004 / 05$ & NS $0,12 \mathrm{a}$ & $0,10 \mathrm{ab}$ & $0,09 \mathrm{a}$ & $0,08 \mathrm{~b}$ & $0,07 \mathrm{ab}$ & $0,09 \mathrm{a}$ \\
\hline $2005 / 06$ & NS $0,02 \mathrm{c}$ & $0,03 \mathrm{c}$ & $0,03 \mathrm{~b}$ & $0,03 \mathrm{c}$ & $0,03 \mathrm{~b}$ & $0,02 \mathrm{~b}$ \\
\hline $2006 / 07$ & $\mathrm{AB} 0,11 \mathrm{ab}$ & A $0,13 \mathrm{ab}$ & B $0,07 \mathrm{ab}$ & B $0,07 \mathrm{bc}$ & B $0,07 \mathrm{ab}$ & $\mathrm{AB} 0,07 \mathrm{a}$ \\
\hline Média & 0,07 & 0,09 & 0,06 & 0,08 & 0,07 & 0,07 \\
\hline CV (\%) & \multicolumn{6}{|c|}{12,47} \\
\hline $2002 / 03$ & A 0,14 a & $\mathrm{AB} 0,09 \mathrm{a}$ & B $0,00 \mathrm{ab}^{1}$ & A 0,16 a & $\mathrm{B} 0,00 \mathrm{~b}^{1}$ & B $0,00 b^{1}$ \\
\hline $2003 / 04$ & NS $0,00 b^{1}$ & $0,03 \mathrm{ab}$ & $0,01 \mathrm{ab}$ & $0,00 \mathrm{~b}^{1}$ & $0,01 \mathrm{~b}$ & $0,00 \mathrm{~b}^{1}$ \\
\hline $2004 / 05$ & NS 0,02 b & $0,00 b^{1}$ & $0,00 \mathrm{~b}^{1}$ & $0,01 \mathrm{~b}$ & $0,01 \mathrm{~b}$ & $0,01 \mathrm{ab}$ \\
\hline $2005 / 06$ & $\mathrm{NS} 0,01 \mathrm{~b}$ & $0,02 \mathrm{ab}$ & $0,02 \mathrm{ab}$ & $0,03 \mathrm{~b}$ & $0,03 \mathrm{ab}$ & $0,01 \mathrm{ab}$ \\
\hline $2006 / 07$ & NS $0,06 \mathrm{ab}$ & $0,07 \mathrm{ab}$ & $0,08 \mathrm{a}$ & $0,06 \mathrm{ab}$ & $0,10 \mathrm{a}$ & $0,08 \mathrm{a}$ \\
\hline Média & 0,05 & 0,04 & 0,02 & 0,05 & 0,03 & 0,02 \\
\hline CV $(\%)$ & \multicolumn{6}{|c|}{25,83} \\
\hline $2002 / 03$ & B $2,24 \mathrm{bc}$ & B 2,18 bc & B $2,31 \mathrm{~b}$ & $\mathrm{AB} 2,65 \mathrm{~b}$ & $\mathrm{AB} 2,55 \mathrm{~b}$ & A $3,29 \mathrm{~b}$ \\
\hline $2003 / 04$ & NS 2,46 bc & $2,68 \mathrm{~b}$ & $2,20 \mathrm{~b}$ & $2,38 \mathrm{bc}$ & $2,69 \mathrm{~b}$ & $2,36 \mathrm{~cd}$ \\
\hline $2004 / 05$ & NS 2,73 b & $2,93 \mathrm{~b}$ & $2,71 \mathrm{~b}$ & $2,25 \mathrm{bc}$ & $2,46 \mathrm{bc}$ & $2,64 b c$ \\
\hline $2005 / 06$ & C 5,89 a & $\mathrm{BC} 6,45 \mathrm{a}$ & B $7,01 \mathrm{a}$ & B 7,12 a & B $7,00 \mathrm{a}$ & A 8,40 a \\
\hline $2006 / 07$ & NS $1,74 \mathrm{c}$ & $1,53 \mathrm{c}$ & $1,31 \mathrm{c}$ & $1,55 \mathrm{c}$ & $1,65 \mathrm{c}$ & $1,62 \mathrm{~d}$ \\
\hline Média & 3,01 & 3,15 & 3,11 & 3,32 & 3,27 & 3,66 \\
\hline CV (\%) & \multicolumn{6}{|c|}{15,38} \\
\hline $2002 / 03$ & $\mathrm{NS} 3,37 \mathrm{~b}$ & $3,18 \mathrm{~b}$ & $3,22 \mathrm{~b}$ & $3,81 \mathrm{~b}$ & $3,35 \mathrm{~b}$ & $2,78 \mathrm{~b}$ \\
\hline $2003 / 04$ & NS 2,66 b & $3,51 \mathrm{~b}$ & $2,41 \mathrm{~b}$ & $3,38 \mathrm{~b}$ & $2,58 \mathrm{~b}$ & $4,07 \mathrm{~b}$ \\
\hline $2004 / 05$ & $\mathrm{NS} 3,73 \mathrm{~b}$ & $3,93 \mathrm{~b}$ & $3,22 \mathrm{~b}$ & $2,69 \mathrm{~b}$ & $3,01 \mathrm{~b}$ & $2,86 \mathrm{~b}$ \\
\hline $2005 / 06$ & B 8,05 a & B 9,67 a & B $10,01 \mathrm{a}$ & A $12,82 \mathrm{a}$ & A $12,71 \mathrm{a}$ & A 14,81 a \\
\hline $2006 / 07$ & $\mathrm{NS} 3,26 \mathrm{~b}$ & $3,55 \mathrm{~b}$ & $2,69 \mathrm{~b}$ & $2,79 \mathrm{~b}$ & $2,41 \mathrm{~b}$ & $2,54 \mathrm{~b}$ \\
\hline Média & 4,21 & 4,77 & 4,31 & 5,10 & 4,81 & 5,41 \\
\hline CV (\%) & \multicolumn{6}{|c|}{26,54} \\
\hline $2002 / 03$ & B $5,83 \mathrm{~b}$ & $\mathrm{AB} 6,26 \mathrm{ab}$ & B 5,70 b & $\mathrm{AB} 6,26 \mathrm{~b}$ & A 6,55 bc & A $6,44 \mathrm{ab}$ \\
\hline $2003 / 04$ & NS 5,93 b & $5,99 \mathrm{bc}$ & $6,08 \mathrm{ab}$ & $6,26 \mathrm{~b}$ & $6,46 \mathrm{c}$ & $6,23 \mathrm{bc}$ \\
\hline $2004 / 05$ & NS 6,22 ab & $5,62 \mathrm{c}$ & $6,11 \mathrm{ab}$ & $5,89 \mathrm{~b}$ & $6,17 \mathrm{c}$ & $5,84 \mathrm{c}$ \\
\hline $2005 / 06$ & B $6,28 \mathrm{ab}$ & B 6,60 a & B 6,40 a & $\mathrm{AB} 6,85 \mathrm{a}$ & A 7,33 a & $\mathrm{AB} 6,76 \mathrm{ab}$ \\
\hline $2006 / 07$ & NS 6,62 a & $6,50 \mathrm{ab}$ & $6,57 \mathrm{a}$ & $6,88 \mathrm{a}$ & $7,08 \mathrm{ab}$ & $6,98 \mathrm{a}$ \\
\hline Média & 6,18 & 6,09 & 6,17 & 6,42 & 6,72 & 6,45 \\
\hline CV (\%) & \multicolumn{6}{|c|}{5,22} \\
\hline $2002 / 03$ & $\mathrm{AB} 48,46 \mathrm{~b}$ & $\mathrm{AB} 58,54 \mathrm{~b}$ & B $42,78 \mathrm{~b}$ & $\mathrm{AB} 56,70 \mathrm{c}$ & A 66,42 bc & A $66,54 \mathrm{~cd}$ \\
\hline $2003 / 04$ & B $45,66 \mathrm{~b}$ & B $57,11 \mathrm{~b}$ & B $47,86 \mathrm{~b}$ & B 59,63 bc & B $47,72 \mathrm{c}$ & A 83,70 bc \\
\hline $2004 / 05$ & NS 59,96 ab & $59,54 \mathrm{~b}$ & $57,56 \mathrm{~b}$ & $52,27 \mathrm{c}$ & $59,79 \mathrm{c}$ & $57,46 \mathrm{~d}$ \\
\hline $2005 / 06$ & $\mathrm{C} 73,32 \mathrm{a}$ & $\mathrm{C} 75,13 \mathrm{~b}$ & BC 84,36 a & BC 79,23 ab & B 96,83 a & A 118,88 a \\
\hline $2006 / 07$ & B $58,30 \mathrm{ab}$ & A 97,36 a & A 87,60 a & A 87,87 a & A $82,98 \mathrm{ab}$ & A 89,68 b \\
\hline Média & 57,14 & 69,53 & 64,03 & 67,14 & 64,22 & 83,25 \\
\hline $\mathrm{CV}(\%)$ & \multicolumn{6}{|c|}{16,73} \\
\hline
\end{tabular}

* Nas linhas, médias não antecedidas da mesma letra maiúscula diferem entre meses; e, nas colunas, médias não seguidas da mesma letra minúscula diferem entre anos pelo teste de Tukey $(\mathrm{P}=0,05)$.

$\left.{ }^{1}\right)$ Não detectado: $<0,006 \mathrm{mg} \mathrm{L}^{-1}$ de $\mathrm{P}$.

${ }^{\text {ns }}$ Teste $\mathrm{F}$ não significativo $(\mathrm{P}=0,05)$. 


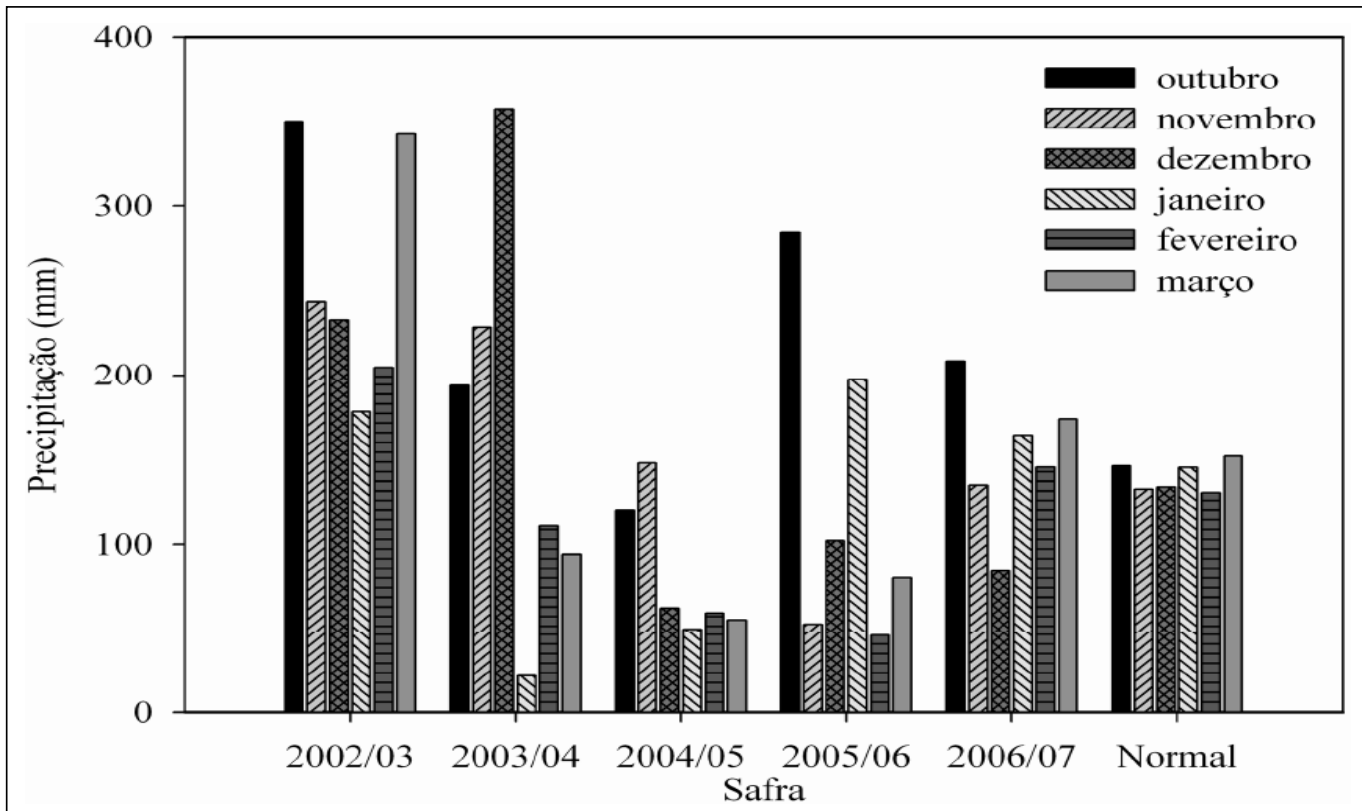

Figura 1 - Precipitação pluvial ocorrida durante os meses de amostragens da água e precipitação pluvial mensa normal em Santa Maria, RS, 2009.

marcantes entre si, provavelmente pelo fato de os rios apresentarem águas correntes. Entretanto, em determinados períodos, o $\mathrm{pH}$ se manteve alcalino, fato que pode estar associado à precipitação pluvial, devido à maior diluição dos compostos dissolvidos (CARVALHO et al., 2000).

Os valores mais elevados da CE da água do rio Vacacaí variaram de 33,38 a $115,51 \mu \mathrm{S} \mathrm{cm}^{-1}$ e do Vacacaí-Mirim de 42,8 a 118,9 $\mu \mathrm{S} \mathrm{cm}^{-1}$ (Tabelas 1 e 2). Nos dois rios, em geral, houve a tendência de aumento desse parâmetro nos meses de novembro, dezembro e janeiro da safra de 2002/03 para a última safra monitorada (2006/07), possivelmente pela redução da precipitação pluvial (Figura 1). Como o rio é normalmente o destino final de todas as águas procedentes da bacia hidrográfica, a elevação da $\mathrm{CE}$ pode estar relacionada ao aumento da temperatura e de sólidos em suspensão na água transferidos das lavouras existentes na bacia hidrográfica para os rios (CARVALHO et al., 2000). Esse fato coincidiu também com a redução no volume de água desses rios em razão das diversas demandas de uso, bem como com a elevada concentração de $\mathrm{Na}$ em 2005/06 (DIEL et al., 2007). Por outro lado, nos períodos com maior precipitação pluvial, foram encontrados os menores valores de CE: $33,33 \mu \mathrm{S} \mathrm{cm}^{-1}$ (rio Vacacaí) e $42,78 \mu \mathrm{S} \mathrm{cm}^{-1}$ (rio Vacacaí-Mirim), consequência da diluição de substâncias orgânicas degradáveis após as chuvas.
O controle da transferência de minerais da bacia hidrográfica para os rios necessita de medidas que reduzam os efeitos do fator fonte e do transporte. Assim, pode-se considerar que a aplicação de fertilizantes em taxa condizente com a demanda de nutrientes pelas culturas e em períodos não coincidente com o alto fluxo superficial favorecerá o controle das perdas decorrentes dos efeitos das variáveis do fator fonte (SHARPLEY et al., 2001). Para o controle das perdas de nutrientes decorrentes da transferência com o fluxo superficial, é necessário impedir o seu transporte, favorecendo a infiltração no solo.

\section{CONCLUSÕES}

O pH variou de 5,71 a 7,50, e a CE, de 33,33 a $118,88 \mu \mathrm{S} \mathrm{cm}^{-1}$. Para N-nitrato, as amostras de água estiveram dentro de limites aceitáveis estabelecidos pelas legislações. A concentração de $\mathrm{P}$ oscilou de 0,00 a $0,18 \mathrm{mg} \mathrm{L}^{-1}$. Os níveis de $\mathrm{N}$-amônia variaram de 0,02 a $0,39 \mathrm{mg} \mathrm{L}^{-1}$, o nível de $\mathrm{K}$, de 2,05 a 3,66 $\mathrm{mg} \mathrm{L}^{-1}$, e o nível de $\mathrm{Na}$, de 1,84 a $16,81 \mathrm{mg} \mathrm{L}^{-1}$

\section{REFERÊNCIAS}

APHA - American Public Health Association. Standard methods for examination of water and wastewater. 18.ed. Sprinfield: Bru-El Graphic, 1995. 368p.

BERTOL, I. et al. Perdas de fósforo e potássio por erosão hídrica em um inceptisol sob chuva natural. Revista Brasileira da 
Ciência do Solo, v.28, n.3, p.485-494, 2004. Disponível em: $<$ http://www.scielo.br/scielo.php?script=sci_arttext\&pid=S0100$06832004000300010 \& \operatorname{lng}=\mathrm{en} \& \mathrm{nrm}=\mathrm{iso} \& \mathrm{t} \ln \mathrm{g}=\mathrm{pt}>$. Acesso em: 29 mar. 2009. doi: 10.1590/S0100-06832004000300010.

BRASIL - CONAMA. Conselho Nacional de Meio Ambiente. Resolução CONAMA n.357 de 17 de março de 2005. Brasília: Diário Oficial da República Federativa do Brasil, 2005. $23 \mathrm{p}$.

BRASIL - Ministério da Saúde, portaria n.518, 25 de março de 2004. Estabelece os procedimentos e responsabilidades relativos ao controle e vigilância da qualidade da água para consumo humano e seu padrão de potabilidade e dá outras providências. $15 \mathrm{p}$.

CARPENTER, S.R. et al. Nonpoint pollution of surface waters with phosphorus and nitrogen. Ecological Applications, v.8, n.3, p.559-568, 1998. Disponível em: $<$ http://www.esajournals.org/doi/abs/10.1890/10510761(1998)008\%5B0559\%3ANPOSWW\%5D2.0.CO\%3B2>. Acesso em: 29 mar. 2009. doi: 10.1890/10510761(1998)008[0559:NPOSWW]2.0.CO;2.

CARVALHO, A.R. et al. Relações da atividade agropecuária com parâmetros físicos químicos da água. Química Nova, v.23, n.5, p.618-622, 2000. Disponível em: <http:// www.scielo.br/scielo.php?script $=$ sci_arttext\&pid $=$ S0 100 40422000000500009>. Acesso em: 29 mar. 2009. doi: $10.1590 / \mathrm{S} 0100-40422000000500009$.

CHAU, K.W. Integrated water quality management in Tolo Harbour, Hong Kong: a case study. Journal of Cleaner Production, v.15, n.16, p.1568-1572, 2007. Disponível em: <http://www.sciencedirect.com/ science?_ob=MImg\&_imagekey=B6VFX-4M4CMY2-2$5 \&$ \& di $=6022 \&$ \& user $=687358 \&$ \&orig $=$ search $\&$ _coverD ate $=11 \% 2 \mathrm{~F} 30 \% 2 \mathrm{~F} 2007 \&$ sk $=999849983 \&$ view $=\mathrm{c} \& w \mathrm{wh}=\overline{\mathrm{d}} \mathrm{GLbVzb}-$ zSkzS\&md5=6c501baf06fc2573698e8047194e6190\&ie=/ sdarticle.pdf $>$. Acesso em: 29 mar. 2009. doi:10.1016/ j.jclepro.2006.07.047.

CORRELL, D.L. The role of phosphorus in the eutrophication of receiving waters. Journal Environmental Quality, v.27, n.2, p.261-266, 1998 .

DIEL, M. et al. Nutrientes na água para irrigação de arroz na Região Sul do Rio Grande do Sul, Brasil. Ciência Rural, v.37, n.1, p.102-109, 2007. Disponível em: <http://www.scielo.br/ pdf/cr/v37n1/a17v37n1.pdf>. Acesso em: 29 mar. 2009. doi: 10.1590/S0103-84782007000100017.

EPA - United States Environmental Protection Agency. Drinking water contaminants. Disponível em: http:// www.epa.gov/safewater/contaminants/index.html\#mcls/. Acesso em: 01 fevereiro de 2008 .

GINTING, D. et al. Interaction between manure and tillage system on phosphorus uptake and runoff losses. Journal Environmental Quality, v.27, n.6, p.1403-1410, 1998.

HAMER, A.J. et al. Amphibian decline and fertilizers used on agricultural land in south-eastern Australia. Agriculture, Ecosystems \&
Environment, v.102, n.3, p. 299-305. 2004. Disponível em: <http:/ www.sciencedirect.com/science?_ob=ArticleURL\&_udi=B6T3Y4B8KBGW-1\&_user $=687358 \&$ rdoc $=1 \&$ fmt $=$ \&_orig=- $=a r c h \&$ sort $=\mathrm{d}$ $\&$ vie w $=$ c \&_a c c t $=$ C $000037899 \&$ version $=1$ $\&$ _urlversion $=0 \&$ _us erid $=687358 \&$ m d $5=5$ a2077e6839c7266065750b59d207be7>. Acesso em: 29 mar. 2009. doi:10.1016/j.agee.2003.09.027.

MARQUES, M.N. et al. Avaliação do impacto da agricultura em áreas de proteção ambiental, pertencentes à bacia Hidrográfica do Rio Ribeira de Iguape (SP). Química Nova, v.30, n.5, p.1171-1178, 2007. Disponível em: <http:// w w w. s c i e 1 o.b r / s c i e 1 o.p h p ? p i d = S 0100 40422007000500023\&script $=$ sci_arttext $>$. Acesso em: 29 mar. 2009. doi: $10.1590 / \mathrm{S} 0100-40 \overline{4} 22007000500023$.

ODEMIS, B.; EVRENDILEK, F. Monitoring water quality and quantity of national watersheds in Turkey. Environmental Monitoring Assessent, v.133, n.1-3, p.215-229, 2007. Disponível em: <http://www.springerlink.com/content/ 2k45h56468234240/>. Acesso em: 29 mar. 2009. doi: 10.1007/ s 10661-006-9574-1.

PINHEIRO, A.; DESCHAMPS, F.C. Transporte de ortofosfato e de nitrato na microbacia do Ribeirão Fortuna, SC. Engenharia Agrícola e Ambiental, v.12, n.3, p.318-325, 2008. Disponível em: <http:/ /www.scielo.br/scielo.php?script $=$ sci_arttext\&pid $=\mathrm{S} 1415$ 43662008000300014>. Acesso em: 29 mar. 2009. doi: 10.1590/ S1415-43662008000300014.

PIONKE, H.B. et al. Critical source area controls on water quality in an agricultural watershed located in de Chesapeake basin. Ecological Engineering, v.14, n.4, p.325-335, 2000.

PIVELI, R.P.; KATO, M.T. Qualidade das águas e poluição: aspectos físico-químicos. São Paulo: ABESAssociação Brasileira de Engenharia Sanitária e Ambiental, 2006. Cap.6 e 7.

RS - Governo do Estado. Secretaria Estadual do Meio Ambiente. Relatório Anual sobre a Situação dos Recursos Hídricos no Estado do Rio Grande do Sul. Porto Alegre: SEMA, 2007. Disponível no site: http://www.sema.rs.gov.br/sema/html/ pdf/Relatorio\%20Anual_completo_210207.pdf. Acesso em: 25 de outubro de 2008 .

RS - Secretaria da Saúde e Meio Ambiente (SSMA). Portaria n.05/89 de 16 de Março de 1989. Aprova a Norma Técnica que dispõe sobre critérios e efluentes líquidos. Diário Oficial, Porto Alegre, 29 de março de 1989.

SHARPLEY, A.N. et al. Phosphorus loss from land to water: integrating agricultural and environmental management. Plant and Soil, v.237, n.2, p.287-307, 2001.

SUNDARAY, S.K. et al. Behaviour and distribution pattern of nutrients in river-estuarine waters of Mahanadi, Orissa, India. Asian Journal of Water, Environmental and Pollution, v.2, n.1, p.77-84, 2005.

TEDESCO, M.J. et al. Análise de solo, plantas e outros materiais. 2.ed. Porto Alegre: Departamento de Solos, UFRGS, 1995. 174p. 